\title{
Enhanced Hand Manipulation Methods for Efficient and Precise Positioning and Release of Virtual Objects
}

\author{
Noritaka OSAWA $^{\dagger \mathrm{a})}$, Member
}

\begin{abstract}
SUMMARY Automatic adjustment methods for efficient, precise positioning and release of a virtual 3D object by direct hand manipulation in an immersive virtual reality environment are described and evaluated. The proposed methods are release adjustment, position adjustment, viewpoint adjustment, and virtual hand size adjustment. Combining these methods enables users to manipulate a virtual object efficiently and precisely. An experimental evaluation showed that these methods were effective and useful in terms of the number of task completions and the subjective preference, particularly for a small virtual target.

key words: direct manipulation by hand, position adjustment, viewpoint adjustment, release adjustment, immersive environment
\end{abstract}

\section{Introduction}

An immersive virtual environment using direct manipulation by hand could offer a novice user a more familiar, simpler, and more efficient way to manipulate a virtual object because a metaphor of hand manipulation in the physical world is presumed to be understood by anyone. For example, changing the position of a virtual object through hand manipulation in an immersive space is similar to manipulating an actual object in the real world, so less specialized knowledge and fewer technical skills are required of a user.

Suppose we want students in a class to experience and understand a lesson through interaction using an immersive virtual environment for hands-on learning. Most such students would be novice users of immersive virtual environments and unfamiliar with 3D pointing devices. Generally speaking, it takes some time to master the use of 3D pointing devices. This could make it difficult for students to use the virtual environment, because the long training time would decrease the time that could be used for hands-on learning within a fixed course period. If the students could not manipulate a virtual object or a virtual space as they like, the hands-on learning would be ineffective. For wide acceptance and effective use of immersive virtual environments, it is thus necessary for casual users such as students to be able to manipulate virtual objects and virtual space precisely and efficiently, without long training. Therefore, we think that direct manipulation by hand, without using a handheld device, is important for novice or casual users, because direct manipulation by hand is more familiar.

While hand manipulation is easy to understand and ap-

Manuscript received September 18, 2007.

Manuscript revised May 21, 2008.

${ }^{\dagger}$ The author is with the National Institute of Multimedia Education, Chiba-shi, 261-0005 Japan.

a) E-mail: osawa@ nime.ac.jp

DOI: 10.1093/ietisy/e91-d.10.2503 ply for approximate positioning, it has been considered unsuitable for making precise adjustments to virtual objects in an immersive environment, because of the difficulties of holding the hand still in midair without support and of releasing an object at a fixed position. We believe, however, that direct hand interaction techniques should be investigated in order to provide effective, intuitive interfaces for immersive virtual environments.

This paper proposes and describes enhanced hand manipulation methods. These methods use automatic adjustments to allow efficient, precise positioning and release of virtual objects. The methods improve the accuracy of hand manipulation without the need for a long training time. Our previous paper has already briefly reported the methods and results of experiments with these adjustment methods [17], [18]. This paper fully describes the adjustment methods and reports on the experiments, in which both the number of tasks completed within a specific period and the completion ratio were measured. The results show that the proposed methods are effective and useful for manipulating a virtual object precisely and efficiently.

\section{Related Work}

Several prototype tools and frameworks enabling users to manipulate objects in a virtual environment have been developed and reported [2], [4], [9], [12], [13], [21]-[23]. They enable objects to be manipulated by hand or with a $3 \mathrm{D}$ pointing device such as the cubic mouse [7].

Moreover, many studies have been done on 3D interaction techniques using hand manipulation, such as the silk cursor [24], ray-casting interaction [3], go-go interaction [20], IntenSelect [8], body-relative interaction [14], image plane interaction [19], and scaled manipulation [6]. These techniques use a handheld device such as a stylus to manipulate a virtual object.

The silk cursor is a 3D target selection technique using semi-transparent surfaces. Ray-casting interaction is a technique to pick up objects by pointing at them. Go-go interaction uses a virtual rubber arm that enables reaching out to and manipulating distant objects. IntenSelect is a selection technique in which the selection ray is effectively snapped to the object by using a selection-by-volume technique. These techniques are mainly for selecting objects, not manipulating them. In contrast, our enhanced hand manipulation methods are for placing a virtual object at a desired position accurately. 
Body-relative interaction is based on proprioception, that is, a person's sense of the position and orientation of his body and its parts. One form of body-relative interaction includes automatic scaling of the world. This is similar to our methods, but the purpose is different. Body-relative interaction uses automatic scaling for manipulating objects outside the user's reach, whereas our methods use automatic scaling for manipulating objects precisely.

Image plane interaction allows the user to interact with the $2 \mathrm{D}$ projections that $3 \mathrm{D}$ objects in the scene make on his/her image. Although image plane interaction techniques can be used for object selection, object manipulation, and user navigation, the user has to select an appropriate mode for his/her purpose. This complicates the use of the techniques for novice users.

Using handheld devices is feasible, but handheld 3D pointing devices might require practice and restrict body movement in an immersive virtual environment, and such restrictions should be avoided. As briefly mentioned above, direct manipulation by hand is more similar to real-life actions than manipulation using a 3D pointing device. Here, direct manipulation by hand means that a user pinches a virtual object between the thumb and fingers to hold it and then opens the hand to release it. This is referred to as direct hand manipulation in this paper. In direct hand manipulation, hand gestures with the fingers can easily be used to give commands to a virtual system. It is difficult to use such hand gestures when holding a device.

This study focused on adjustment methods for direct hand manipulation; it was not concerned with specific devices to capture motions of the hand and fingers, although we think that a camera array system or a camera-based optical motion capture system would be useful for capturing hand and finger motions because the user would not have to wear a device such as a sensor glove. Moreover, a camerabased system allows users to reduce the time to put on and take off motion capture devices such as sensor gloves. This is especially useful for a hands-on learning in a class as described in the introduction. Camera-based motion capture systems have been used (e.g. [11]), but they cannot give tactile feedback to the user. Because interaction methods should be effective in using camera-based motion capture systems, we did not give force or tactile feedback to the user, although such feedback could improve the ease of manipulation tasks. At present, there is no optical camera-based motion capture system that can precisely recognize small finger motions without markers is readily available. Hence, a sensor glove was used to capture the motions of the hand and fingers. A future study will use an advanced camerabased optical motion capture system.

Moreover this paper focuses on positioning and release of virtual objects. Grabbing of an object can be difficult in an environment filled with virtual objects. However, this is true for other methods using 3D devices as well as direct hand manipulation. This research studied methods to overcome the weak points of direct hand manipulation.

\section{Automatic Adjustments}

Two difficulties arise with direct hand manipulation after grabbing a virtual object. One is the difficulty of moving a virtual object to a precise position (precise positioning), while the other is the difficulty of releasing it at a precise position (precise release). Precise positioning requires accurate position control and precise release requires accurate release timing. As noted above, it is not easy for people to hold a hand precisely in midair without physical support. It is also hard to release a virtual object in a precise position because the releasing action often causes the hand to move.

The proposed automatic adjustments include release adjustment, position adjustment, viewpoint adjustment, and virtual hand size adjustment, which is referred to simply as size adjustment. The release adjustment adjusts the release timing for precise release. The position and viewpoint adjustments adjust the virtual hand position and the viewpoint, respectively, for precise positioning. The size adjustment is used with the viewpoint adjustment to keep the apparent virtual hand size constant. The details of these adjustments are described in the following sections.

These adjustments are based on speed. The release adjustment is based on the relative speed between the tips of the thumb and the pinching finger. The position and viewpoint adjustments are based on hand speed. One assumption underlying the proposed adjustments for precise positioning is that the hand moves slowly when the user wants to manipulate a virtual object precisely. PRISM [6] also uses speed-dependent techniques but controls only the position, whereas the proposed methods here control not only the position but also the viewpoint, the size, and the release location. Moreover, the adjustment model for PRISM is different from that of the stage model proposed here and described in Sect. 8.1.

\section{Precise Release}

In previous experiments without the release adjustment [16], some of the subjects opened their hands quickly to release a virtual object at a fixed position. This action can be used as an event to estimate the true timing of the release. The release adjustment method exploits this action.

When a virtual object is quickly released-that is, when the thumb and fingers are opened quickly-the virtual object is returned to the position that seems to be the intended release position.

This adjustment is based on the reaction time needed to respond to a stimulus, that is, the stimulus-response reaction time. According to the information processing model by Card et al. [5], it takes time for a user to recognize a virtual object's position, to understand that he should release the virtual object, and to actuate his muscles to release it.

When a virtual object is to be released from the hand, the most appropriate release position is sought. To recognize the release action, the relative speed of the thumb and 
the pinching finger is measured. This is referred to as the release speed. Let $T_{s}$ be the point in time when the release speed becomes faster than the triggering speed $S_{t}$. In the research reported here, $S_{t}$ was set to be a constant $10 \mathrm{~mm} / \mathrm{s}$. Our pilot study showed that about $92 \%$ of the relative speed of the thumb and the pinching finger during a precise positioning task were slower than $10 \mathrm{~mm} / \mathrm{s}$. Since $S_{t}$ was measured in a position-tracker space, i.e., a physical space, not a virtual space, the virtual hand size adjustment and the viewpoint adjustment do not affect its value. Let $T_{r}$ be the point when the release is detected by the system. The system detects the release when the thumb and/or a finger do not collide with a virtual object and the distance between the thumb and finger is longer by a constant $(20 \mathrm{~mm})$ than the distance at which the thumb and finger pick up the virtual object.

All data samples from the sensor glove and position tracker are saved with timestamps in a data sample history buffer. A candidate data sample for an appropriate release position, i.e., an adjusted position, can be found from $\left(T_{s^{-}}\right.$ $\left.T_{\min }\right)$ to $\left(T_{r}-T_{\max }\right)$. Because the stimulus-response time is greater than zero, there is no candidate data for an appropriate release position during some period before $T_{s} . T_{s}-T_{\min }$ is such a period in which there is no candidate data. $T_{\min }$ is determined by considering the stimulus-response reaction time, system overhead, and latency. The preliminary experiment used $300 \mathrm{~ms}$ as $T_{\min }$. The second experiment set $200 \mathrm{~ms}$ as the value of $T_{\min }$ because the software processing had been improved and to extend the search range. The search for the appropriate release position is limited to $T_{\max }$ before the release $T_{r}$, which restricts the buffer size of the data sample history. Old unused data are discarded from the data sample history buffer. This research used a value of $1000 \mathrm{~ms}$ as $T_{\max }$. Figure 1 shows the relationship between the estimated release timing and a release action. If an appropriate release position is not found within the search period, the position at $T_{r}$ is used as the release position.

In estimating the true release timing, a simple time offset with respect to the release action detection was not used. This is because the duration of a release action may depend on the individual; that is, the period between the true release timing and the timing when the system recognizes the release can vary among people. Therefore, the relative speed between the thumb and the finger was chosen as the trigger for detecting the release.

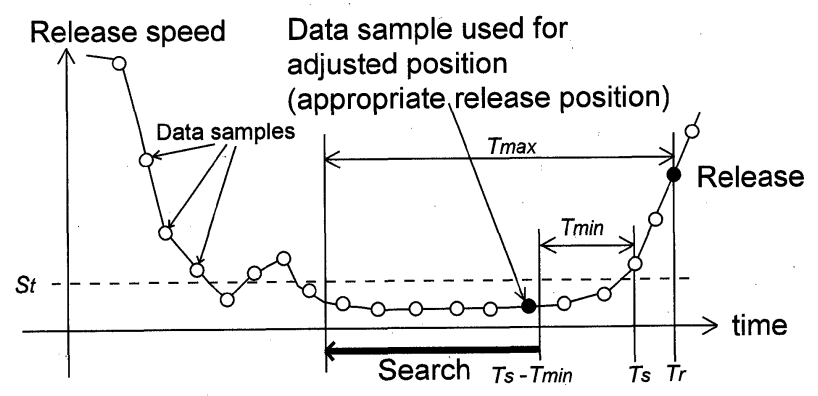

Fig. 1 Release adjustment.

\section{Precise Positioning}

Precise positioning uses the position, viewpoint, and size adjustments, which are controlled by scale factors. This section describes the basic mechanisms of these adjustments. The specific control models used for the adjustments are described in sections covering the experiments, each of which used a different model.

\subsection{Position Adjustment}

The position adjustment method is designed to enable precise positioning when the hand moves slowly. This method adjusts the position of the virtual hand. The adjusted position $P_{a}$ is expressed by

$$
P_{a}=P_{a}^{\prime}+F_{d}\left(P_{m}-P_{a}^{\prime}\right)+F_{r}\left(P_{m}-P_{m}^{\prime}\right)
$$

where $P_{a}^{\prime}$ is the adjusted position at the last calculation, $P_{m}$ is the measured hand position, $P_{m}^{\prime}$ is the hand position at the last measurement, $F_{d}$ is a displacement scale factor (or offset recovery scale factor), and $F_{r}$ is a relative position scale factor. $F_{r}$ is used to reduce the movement of the hand in the precise positioning, while $F_{d}$ is used to recover the offset between the adjusted and measured positions. The relationships between the measured and adjusted positions are shown in Fig. 2.

\subsection{Viewpoint and Size Adjustment}

The viewpoint and size adjustment methods are also used for precise positioning when the hand speed is slow. The viewpoint scale factor $F_{v}$ and the virtual hand size scale factor $F_{s}$ control these adjustments.

When the viewpoint is not adjusted, $F_{v}$ equals 1 . As $F_{v}$ is decreased, the viewpoint approaches the point where the virtual object is grasped (the grabbing point). This movement of the viewpoint enlarges the scene containing the virtual object. Figure 3 illustrates the viewpoint adjustment.

Enlargement is useful for precise positioning when a user can control his hand precisely. Enlargement for precise manipulation is often used in daily life. For example, people often use a magnifying glass to manipulate a precision machine such as a mechanical watch or to do delicate embroidery. Similarly, a surgeon uses a mesoscope for microscopic surgery, and a biologist uses a stereomicroscope

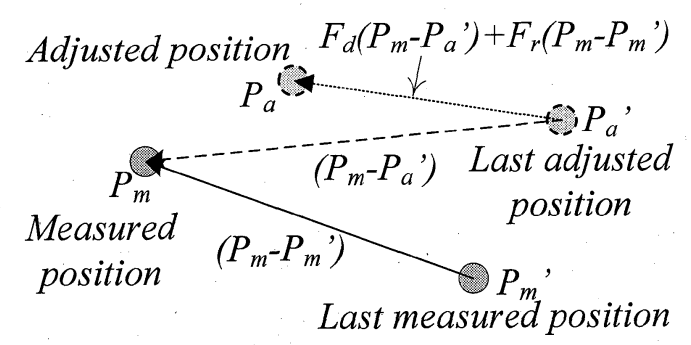

Fig. 2 Relationships between measured positions and adjusted positions. 


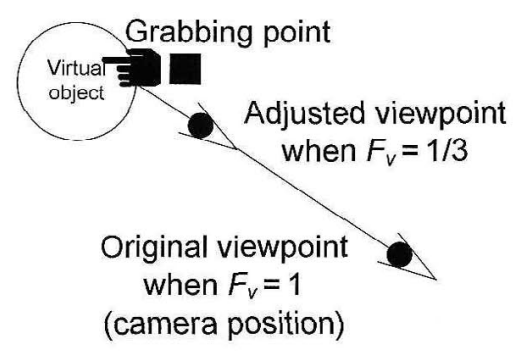

Fig. 3 Viewpoint adjustment.

to cut a small specimen with a knife. In other words, enlargement is useful and important for precise manipulation.

Using only the viewpoint adjustment enlarges the apparent size of the hand, which can prevent the user from seeing the virtual object. Therefore, virtual hand size adjustment is used with the viewpoint adjustment to keep the apparent size of the hand constant. $F_{S}$ controls the virtual hand size and was set equal to $F_{v}$ when the virtual hand size adjustment was used in the experiments. Combining the viewpoint and size adjustments kept the apparent hand size constant.

Speed-dependent zooming techniques have been used in graphical user interfaces (GUIs) [10]. In terms of speed dependence, these techniques are similar to the viewpoint adjustment described here, but the speed control methods are different, and these methods can significantly influence usability. Speed-dependent zooming in a GUI uses a mouse to control the mouse pointer's speed and displacement relative to a base point (usually the center), thus determining the speed. That is, speed-dependent zooming uses rate control. On the other hand, direct hand manipulation uses position control. When using a mouse on a desk, it is easy to keep the speed constant because the speed only changes when the mouse is moved. It is not easy, however, to keep the hand speed constant. It is thus necessary to consider how to implement efficient, useful, speed-dependent viewpoint adjustment.

\section{Experiments}

Two experiments were conducted to clarify the features of the proposed automatic adjustment methods and to evaluate the usefulness of the adjustments for manipulating virtual objects in an immersive environment. The first experiment was preliminary, with the second experiment designed on the basis of the preliminary results. This section describes the common characteristics used in both experiments. The characteristics specific to each experiment are described in the two following sections.

The hypothesis in this research was that the proposed methods would be effective for making precise adjustments efficiently, because they could help adjust the position of an object precisely and release the object appropriately. This hypothesis was tested in terms of performance (completion ratio and average number of completions) and subjective preference.

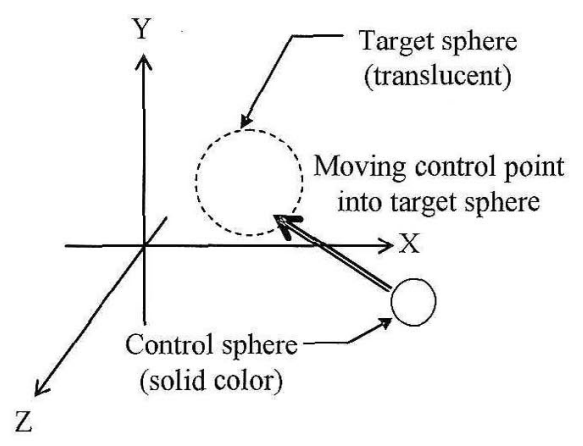

Fig. 4 Basic experimental task.

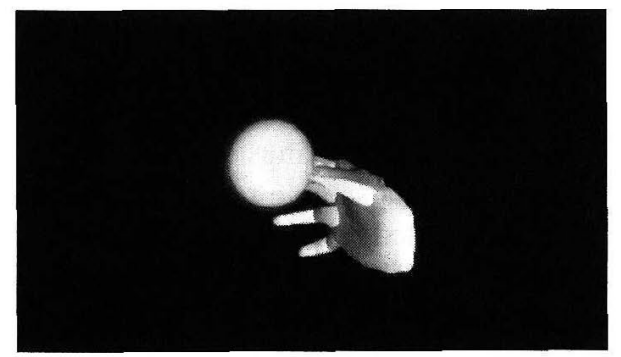

Fig. 5 The control sphere turns aqua when it is moved into the target sphere (with viewpoint and position adjustments).

\subsection{Experimental Environment}

The experiments were done in a virtual environment called TEELeX [1], a kind of surround-display system using immersive projection technology. It has a large cubic screen, where each face measures $3 \mathrm{~m}$ by $3 \mathrm{~m}$. It is a passive stereo system using circular polarization to provide a stereoscopic view. One stereoscopic face was used in these experiments to evaluate the adjustment methods in a simple and usual VR environment.

Both experiments used a system running on a PC workstation (Dell Precision 530 with dual 2-GHz Pentium 4 Xeon processors and a 3DLabs Wildcat II 5110 graphics board supporting dual displays). A six-DoF (degree of freedom) position tracker (Polhemus Fastrak) and sensor gloves (Virtual Technologies CyberGlove) were used to detect the position and motion of the user's hand. The software for the experiments was developed using the Java programming language, the Java 3D class library, and the it $3 \mathrm{~d}$ class library for interactive 3D applications [15].

\subsection{Basic Task}

Figure 4 illustrates the basic task in the experiments. A subject was asked to move a control sphere into a translucent target sphere, and then to release the control sphere within the target sphere. When the control sphere was moved into the target sphere, the control sphere turned aqua, indicating to the subject that it was within the target region. Figure 5 shows a screenshot of the situation when the control sphere has been moved into the target sphere. When the control 
sphere was released outside the target sphere, the control sphere turned pink to indicate that the release was unsuccessful.

The subject stood in an upright stance without physical support. The forefinger and the thumb were used to grasp the virtual object; no other fingers were used.

When adjustment for precise positioning was applied, the virtual hand was colored yellow to explicitly show the status of automatic adjustment. Figure 6 shows screenshots of the situations when automatic adjustments are not applied.

Figure 5 also shows the viewpoint and size adjustments. The target and control spheres are enlarged because the viewpoint has approached the grabbing point. The apparent size of the virtual hand, however, is the same as when no adjustments are used. The user can thus look at the scene minutely, with the viewpoint and size adjustments helping him understand the precise positions of the virtual objects and the virtual hand.

\subsection{Settings}

Sizes and positions are specified by a coordinate system in a position-tracker space, or a physical space. The origin $(0,0,0)$ of the position-tracker space lies at $1200 \mathrm{~mm}$ above the center of the floor screen face.

The radius of the control sphere was $15 \mathrm{~mm}$. The target sphere had a radius of $20 \mathrm{~mm}$ (Size L), $17 \mathrm{~mm}$ (Size M), or $16 \mathrm{~mm}$ (Size S). Our previous study [16] showed that with sizes larger than $20 \mathrm{~mm}$, automatic adjustments are not usually necded to complete the task. The sphere was chosen as the shape of the control and target objects because orientation was not considered in these experiments.

In order to avoid motion sickness in the experiments, a viewpoint camera was placed at a fixed position $(0,0,400)$ (unit: $\mathrm{mm}$ ) and along the $-Z$ axis direction in the positiontracker space where $F_{v}=1$. In other words, head tracking was not employed in the experiment. The angular field of view of the camera was 90 degrees. Both the horizontal field of view and the vertical field of view were the same because the width and height of the screen face was the same as described in Sect. 6.1.

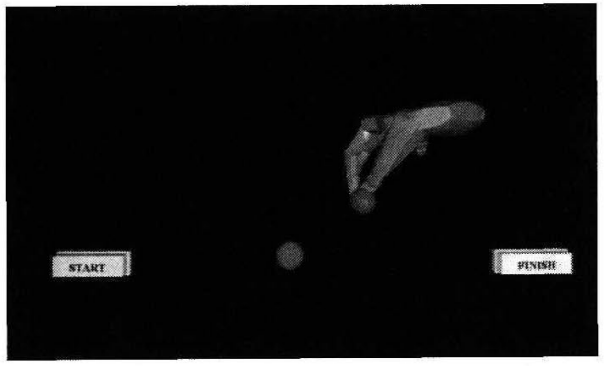

Fig. 6

Virtual hand without adjustments for precise positioning.

\section{Preliminary Experiment}

First, a preliminary experiment was conducted to select important combinations of adjustments.

\subsection{Linear Change Model}

The preliminary experiment used a linear change model to adjust the scale factors. In this model, the scale factors were changed according to a linear relationship with the speed over a control range.

Log records from our previous study showed that about 95\% of the movements during a precise positioning task were made at speeds slower than $5 \mathrm{~mm} / \mathrm{s}$, as shown in Fig. 7 . Therefore, $5 \mathrm{~mm} / \mathrm{s}$ were chosen as a threshold $S_{L}$ for determining slow, precise movements. Moreover, when the speed exceeded $50 \mathrm{~mm} / \mathrm{s}$, precise manipulation generally could not be done. Thus, adjustments for precise positioning were turned off when the hand moved faster than a threshold $S_{H}$ of $50 \mathrm{~mm} / \mathrm{s}$.

The preliminary experiment used the scale factor functions shown in Fig. 8 for the displacement scale factor $F_{d}$, the relative position scale factor $F_{r}$, and the viewpoint scale factor $F_{v}$. As stated previously, when the size adjustment was used, the virtual hand size scale factor $F_{s}$ was the same as $F_{v}$.

When the hand speed was slower than $S_{L}$, the scale factor values were $F_{d}=0, F_{r}=1 / 3$, and $F_{v}=1 / 3$, so

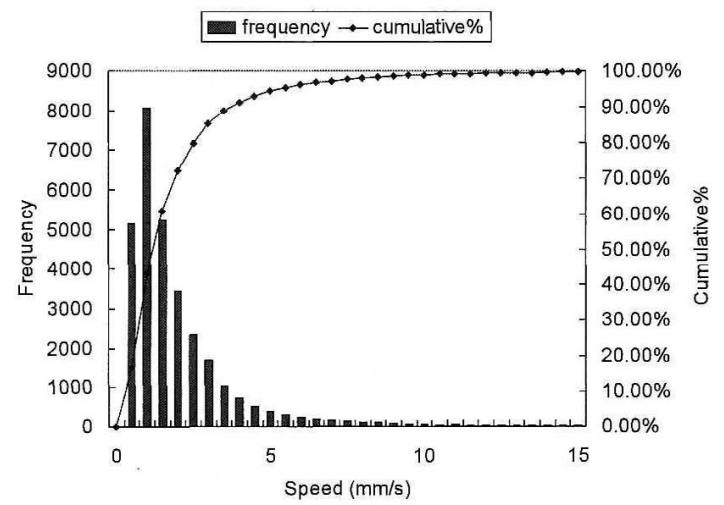

Fig. 7 Distribution of hand speeds during precise manipulation.

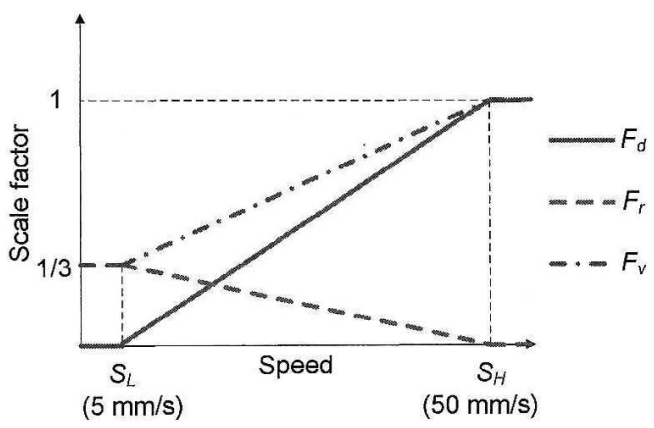

Fig. 8 Scale factor functions used in the linear change model. 
that scaled relative movement, consisting of one-third of the original movement, was accumulated, but displacement recovery was not applied. When the speed was faster than $S_{H}$, the scale factor values were $F_{d}=1, F_{r}=0$, and $F_{\nu}=1$. In this case, the hand position was the same as the measured position, that is, $P_{a}=P_{m}$. When the hand speed was between $S_{L}$ and $S_{H}$, the scale factors changed according to linear relationships with the hand speed, as shown in Fig. 8.

Smaller scale factor values for $F_{r}$ and $F_{v}$ would make it possible to move minutely and to enlarge the scene more, but they might also make it difficult to move coarsely and to see the surroundings of the enlarged region. This could prevent efficient positioning. An appropriate balance between precision and efficiency is essential. Therefore, 1/3 was chosen as the value for $F_{r}$ and $F_{v}$ when the hand speed was slower than $S_{L}$.

\subsection{Combinations of Adjustments}

With position, size, and viewpoint adjustment applied, there are eight possible adjustment combinations: direct manipulation without automatic adjustment (referred to as direct or case 0 ), position adjustment alone (referred to as pos or case 1), hand size adjustment alone (referred to as size or case 2 ), viewpoint adjustment alone (referred to as view or case 4), size +pos (case 3), view+pos (case 5), view+size (case 6), and view+size+pos (case 7). As explained above, the system was designed so that the viewpoint adjustment worked with the size adjustment. Therefore, cases (4) and (5) were omitted from the experiment. In summary, the following six methods were tested: (0) direct, (1) pos, (2) size, (3) size+pos, (6) view + size, and (7) view + size + pos.

Moreover, when release adjustment was used, there were six more combinations: release adjustment only (referred to as rel or case 8), (9) rel+pos, (10) rel+size, (11) rel+size+pos, (14) rel+view+size, and (15) rel+view+size+pos.

\subsection{Task}

One trial was completed when the control sphere was released within the target sphere. In the preliminary experiment, no more tries was attempted as part of one trial.

Although the subjects were asked to complete the task as quickly as possible, some could not complete it within the specified period in some trials. We used a cut-off time of 1 minute, because it is undesirable to take more than this for one instance of precise manipulation. Moreover, we did not want the subjects to become too tired and/or stressful after one trial. When a subject could not complete the task within the cut-off time, the trial was referred to as incomplete.

The control sphere was initially centered at $(50,50,50)$ (unit: $\mathrm{mm}$ ), and the target position was $(0,0,0)$ in the position-tracker space. The target spheres were size $\mathrm{L}$ or M.

\section{$7.4 \quad$ Subjects}

Twelve subjects (seven male, five female) took part in the preliminary experiment. They were all undergraduate students. They had little or no virtual reality experience and were paid for their participation. The subjects performed the task using the various methods in different orders.

\subsection{Procedure}

For each method, the functions of the system and the task were explained to the subjects, and each subject was given practice tasks in order to learn how to use the sensor glove and the interaction methods. These practice sessions were followed by data collection sessions. Each of the 12 subjects tried each method twice, giving a total of 24 trials for each method.

After they finished testing all the methods, the subjects were asked to complete a questionnaire. The question was: "How do you rate the method tested just now? Please rate each item on a scale of 0 to 9 ." In the scale, 0 indicated the lowest preference, and 9 indicated the highest preference. The question referred to six items: speed, accuracy, ease of use, fatigue, satisfaction, and desire to use the method in the future.

\subsection{Completion Ratio}

Figure 9 shows the ratio of completed trials for each method and both target sizes. The figure shows that some methods resulted in incomplete trials, in which the subject did not complete the task within the time limit, especially when the target size was $\mathrm{M}$.

Using the release adjustment method significantly improved the completion ratio when the target size was $\mathbf{M}$. There was a significant difference for each method with and without the release adjustment (all $p<0.05$ with a twotailed Fisher's exact test).

Moreover, when the target size was $\mathrm{M}$, direct was outperformed by all other methods, and there was a significant

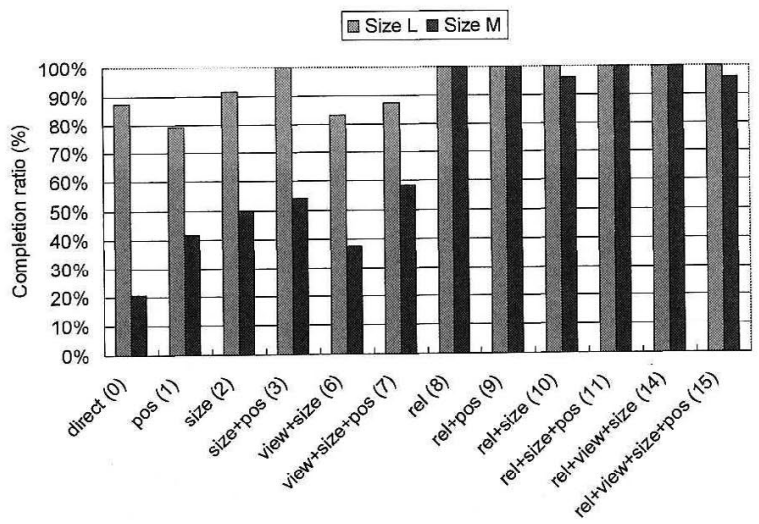

Fig. 9 Completion ratio of trials. 


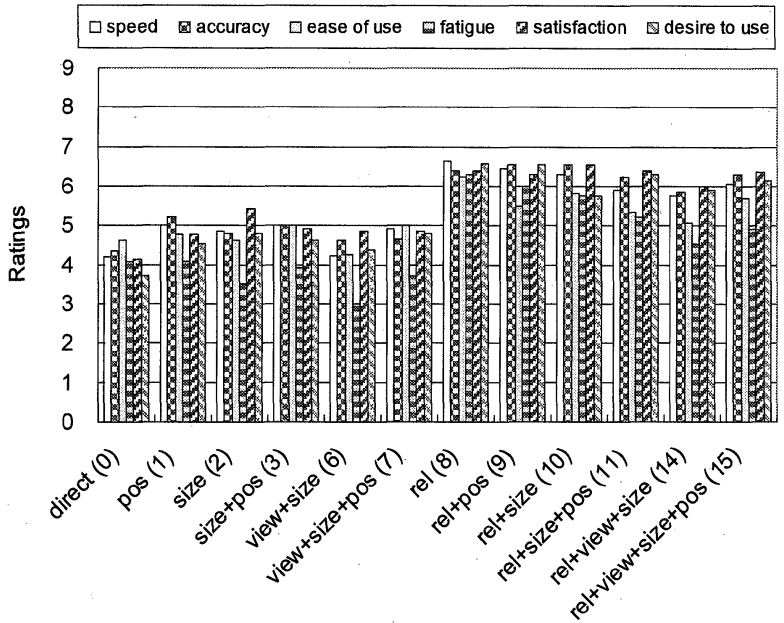

Fig. 10 Specific ratings of subjective preference $(0=$ lowest preference, $9=$ highest preference).

difference in the number of incomplete trials between the (0) direct and (7) view+size+pos methods $(p<0.01$ with a two-tailed Fisher's exact test), between the (0) direct and (3) size +pos methods ( $p<0.05$, same test), and between the $(0)$ direct and $(8,9,10,11,14,15)$ methods with release adjustment (all $p<0.001$, same test).

\subsection{Preferences}

Figures 10 and 11 show the specific ratings for each questionnaire item and the overall subjective rating, respectively. The overall ratings were obtained as the mean values of the answers given by the subjects for the six items.

The methods using release adjustment were rated higher than those that did not use it. An analysis of variance (ANOVA) was applied to analyze the ratings shown in Fig. 11. A significant difference was also found between the overall ratings of the methods with and without release adjustment: $F(1,10)=267.2, p<0.0001$.

\subsection{Results}

The preliminary experiment showed that the release adjustment was the most important of the adjustments. Even if precise positioning is achieved, it is difficult to place a virtual object at a specified position unless the object is released precisely. The results suggested that the release adjustment was followed in importance by the position adjustment, which enables the user to move the virtual object to a specified position. Ranked third was the viewpoint adjustment, which allows the user to look at the target minutely, making it easier to judge whether the virtual object is positioned within the target. As shown in Fig. 9, (6) view+size method was inferior to (1) pos method for size $\mathrm{M}$ without the release adjustment. Moreover the results of the preliminary experiment suggested that the target sizes used were too large to demonstrate the effectiveness of the methods for precise positioning.

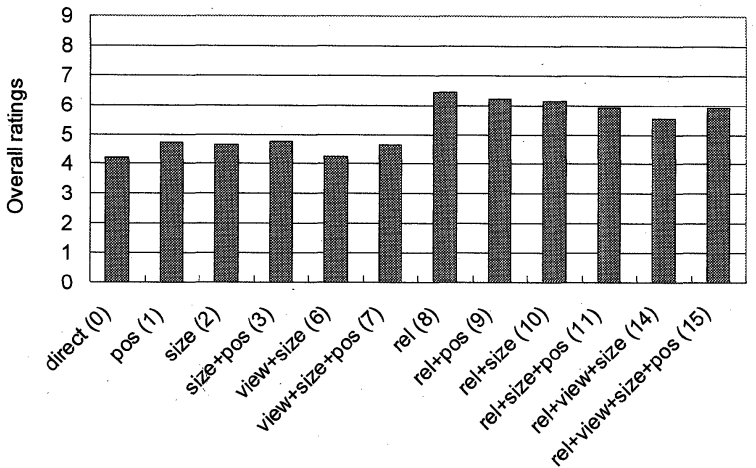

Fig. 11 Overall subjective preference $(0=$ lowest preference, $9=$ highest preference).

\section{Experiment}

On the basis of the preliminary experimental results, this experiment focused on important combinations of the adjustment methods and smaller target sizes. Four methods were chosen for this experiment: (0) direct, (8) rel, (9) $r e l+p o s$, and (15) rel+view+size+pos. The preliminary experiment confirmed that automatic adjustments for precise positioning are usually not needed for target sizes larger than L. Therefore, the four methods were tested with the two smaller target sizes, $\mathrm{M}$ and $\mathrm{S}$.

\subsection{Stage Model with Hysteresis}

In the preliminary experiment, the scale factors for the position, viewpoint, and size adjustments were changed according to linear relationships with the speed over a control range, as shown in Fig. 8. The subjects in the preliminary experiment, however, disliked the linear change of viewpoint, because the scene often changed or was constantly changing. This was a distraction during the manipulation, even though a linear adjustment was performed only while the hand speed was within the specified range. Therefore, a stage model was developed and used in this experiment. In the simplest case, two stages were used, referred to as the precise stage and the normal stage. In the precise stage, or when the hand speed was slow, the adjustments for precise positioning were activated. In the normal stage, i.e., when the speed was fast, these adjustments were deactivated.

\section{(1) Hysteresis}

A simple way to change the stage would be to use a threshold. When the speed exceeds a threshold, the stage would be normal; otherwise, it would be precise. This threshold method is simple but not desirable, because the stage could fluctuate rapidly if the speed is varied near the threshold. To avoid such rapid fluctuation, a form of hysteresis was introduced, requiring two thresholds. In this context, hysteresis means that the stage does not instantly follow the speed but depends on its immediate history. When the speed is below the lower threshold, the precise stage is entered. This 


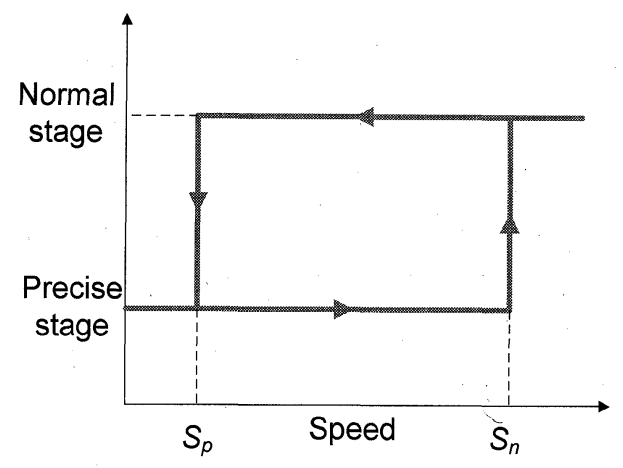

Fig. 12 Stage model with hysteresis.

stage is then maintained until the speed exceeds the higher threshold, at which time the stage is reset to normal.

In the preliminary experiment, the subjects disliked both frequent changes of viewpoint and continuous changes of viewpoint, and some of them tried to avoid viewpoint adjustment. Some of them often prematurely released the virtual object in order to prevent their hand speed from becoming too slow, thus preventing the viewpoint from changing. These avoidance behaviors make viewpoint adjustment useless, so the thresholds were chosen so as to prevent frequent changes of the scale factor. The precise stage should be entered only when precise positioning is needed, and it should be maintained until canceling actions become apparent.

The lower threshold $S_{p}$ and higher threshold $S_{n}$ were set as 1.5 and $300 \mathrm{~mm} / \mathrm{s}$, respectively. Log records from our pilot study showed that about $60 \%$ of the movements during a precise positioning task were made more slowly than $1.5 \mathrm{~mm} / \mathrm{s}$. When the speed was below $S_{p}$, it was rare for precise positioning not to be needed. Therefore, the value of $1.5 \mathrm{~mm} / \mathrm{s}$ was chosen as $S_{p}$. Because the speed rarely exceeded $300 \mathrm{~mm} / \mathrm{s}$ during precise positioning and occasional movements for hand rests, the precise stage could be turned off after the hand moved faster than $300 \mathrm{~mm} / \mathrm{s}$, which was thus chosen as $S_{n}$. The stage model with hysteresis is illustrated in Fig. 12.

As described in Sect. 3, the proposed position adjustment method is similar to that used in PRISM. The speed parameter in PRISM, however, is much faster than the lower threshold speed used here. The lowest parameter (Default Min V) used in the PRISM experiments was $50 \mathrm{~mm} / \mathrm{s}$, whereas the lower threshold in this experiment was $1.5 \mathrm{~mm} / \mathrm{s}$. Figure 7 indicates that position adjustment does not help users adjust the position of a virtual object precisely when the hand speed is faster than $50 \mathrm{~mm} / \mathrm{s}$. The speed parameters in PRISM (which uses a stylus to manipulate the virtual object) are too fast and are inappropriate for direct hand manipulation. Moreover, the proposed adjustments in this experiment used the stage model with hysteresis for scale factor control, whereas PRISM uses a linear change of position control parameters within a control range.

\section{(2) Scale factors and parameters}

In the precise stage, the scale factor values were $F_{d}=0$, $F_{r}=1 / 3$, and $F_{v}=1 / 3$, whereas values of $F_{d}=1, F_{r}=0$, and $F_{v}=1$ were used in the normal stage. These values are similar to those in the linear change model of the previous experiment, but the scale factors were not changed according to linear relationships with the speed.

Some smoothing was adopted, in order to avoid the sudden changes that some subjects disliked when the stage was changed. When viewpoint adjustment was applied, transient animation was used. When the size was changing in the viewpoint adjustment, the maximum rate of change of $F_{v}$ was $2.0 \mathrm{~s}^{-1}$. The transient animation was used only when the virtual object was grasped. When the virtual object was released, the hand size quickly returned to the normal size.

\subsection{Task}

In this experiment, subjects were asked to move a control sphere into a translucent target sphere repeatedly within a specific period. In other words, this experiment measured the number of completions within the specific period. The period of one trial was 1 minute. The period during which the virtual object was grasped was counted as part of the trial period.

The initial positions of the control and target spheres were not fixed but randomly generated within a cubical space whose diagonal vertexes were at $(-150,-150,-150)$ and $(150,150,150)$ (unit: $\mathrm{mm}$ ). When the control sphere was released inside the target sphere and one task was completed, the positions of the control and target spheres were changed for a new task.

\subsection{Subjects}

Fifteen subjects (nine male, six female, all university students) took part in the experiment. They had taken part in our previous experiments using direct hand manipulation in immersive virtual environments although they were still novice users. The subjects were paid for their participation.

\subsection{Procedure}

A procedure similar to that of the preliminary experiment was used. The functions of the system and the task were explained to the subjects, and each subject was given two short practice tasks in order to learn how to use the sensor glove and the interaction methods. These practice sessions were followed by data collection sessions. Using the four methods, the subject performed the task first with a target of size $\mathrm{M}$ and then with a target of size $\mathrm{S}$. This procedure was repeated twice during the data collection sessions.

After they had finished testing each method, the subjects were asked to rate that method on a scale of 1 to 5 (where 1 indicated lowest preference and 5 indicated highest preference) in terms of speed and accuracy, and overall. 


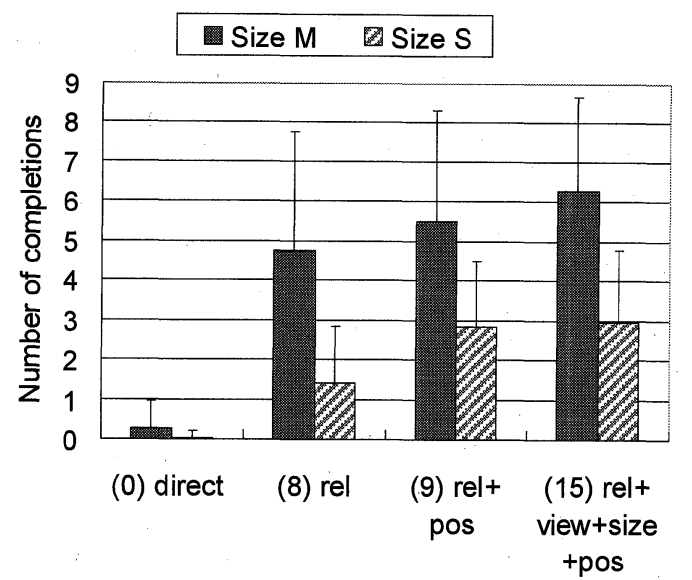

Fig. 13 Average number of completions (with standard deviation error bars).

\subsection{Performance}

Figure 13 shows the average number of completions within the trial period. Using the release adjustment method significantly improved the number of completions. Moreover, the position and viewpoint adjustments improved the number even more. An ANOVA was applied to analyze the number of completions for each method. The ANOVA showed that there were significant differences among the ratings for the (0) direct, (8) rel, (9) rel+pos, and (15) rel+view+size+pos methods: for size $\mathrm{M}, F(3,116)=38.1, p<0.00001$; for size $\mathrm{S}, F(3,116)=27.9, p<0.00001$.

Figure 14 shows the completion ratios, i.e., the ratios of completed trials for each method and target size. This corresponds to Fig. 9 for the preliminary experiment. The number of completions and the completion ratios were derived from the same experimental data. A completed trial means that at least one completion was performed within a specific trial period. This is important in the sense that adjustment can make manipulation possible within a reasonable period, and that it is useful in practice. As shown in the figure, only one trial $(1 / 30$, or about $3.3 \%)$ was completed for size $\mathrm{S}$ with the direct method, whereas all trials (100\%) were completed with at least one success when method (15) rel+view+size+pos was used for size M. A small completion ratio is a problem because it means that many of the subjects could not complete the manipulation within 1 minute. These results show that the adjustment methods were useful for making precise adjustments efficiently.

When the adjustment methods were not used, some subjects could not complete some tasks. Direct hand manipulation could not complete the tasks due to measurement errors of a position-tracker and control errors of hand. The proposed adjustment methods enabled the subjects to complete the tasks within a practical period of time. In real applications, the use of an interface that results in incomplete trials would be impractical. The adjustment methods could improve this situation. In other words, they enhance the reliability to complete a task within a reasonable period of time.

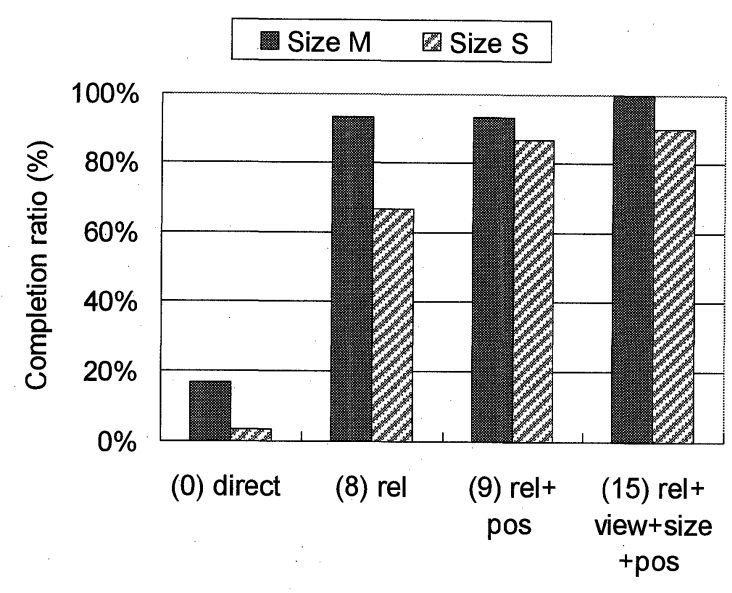

Fig. 14 Completion ratios.

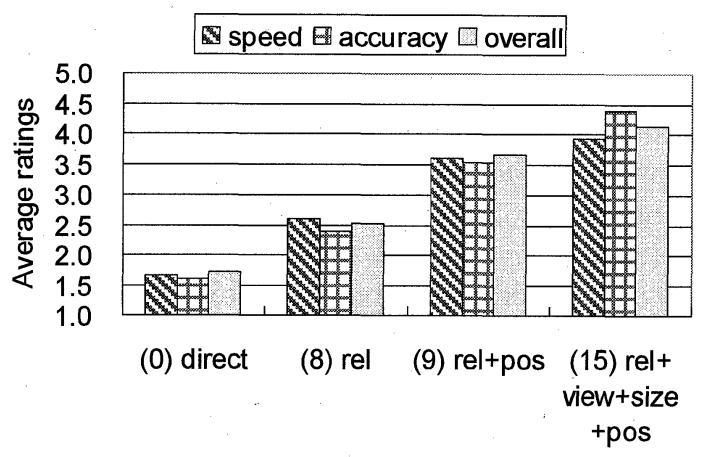

Fig. 15 Specific ratings of subjective preference $(1=$ lowest preference, 5 = highest preference).

\subsection{Preferences}

Figure 15 shows the subjective ratings for this experiment. The methods that used our adjustments were rated higher than the (0) direct method. The (15) rel+view+size+pos method was regarded as best, and the (9) rel+pos method was regarded as second best. These results support the above experimental performance evaluation.

Accuracy was rated higher than speed for the (15) rel+view+size+pos method. This suggests that viewpoint adjustment contributed to accuracy rather than to speed. This is an expected result.

Figure 16 shows the subjective evaluations of the basic adjustment methods. The position adjustment was rated lower than the release and viewpoint adjustments. We think that this is because the effects of the release and viewpoint adjustments were apparent to the subjects, whereas the difference between the position adjustment being on and off could not be distinguished by some of the subjects, besides the visual color feedback. The performance results show, however, that the position adjustment was effective, and the subjective evaluation of the position adjustment was higher than the middle score, (i.e., 3), suggesting a positive impression.

As mentioned before, many subjects in the preliminary 


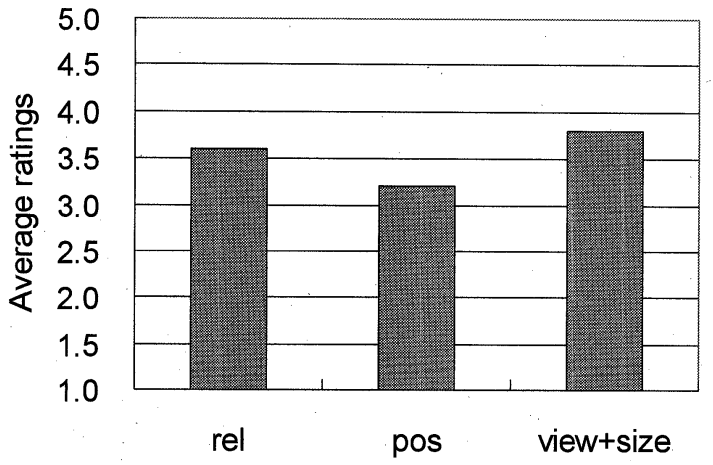

Fig. 16 Subjective evaluation of the basic adjustments.

experiment reported the linear change of viewpoint was distracting. No subjects in this experiment reported that the stage model with hysteresis was a distraction although a few subjects disliked any viewpoint change. This suggests the stage model with hysteresis was better than the linear change model, although the further experiments would be necessary to evaluate the models quantitatively. This preference is partially supported by comparing overall subjective preferences of the (15) rel+view+size+pos method in Figs. 11 and 15. In Fig. 11, the overall preference of (15) can be converted to 3.46 on a five-point scale whereas the value in Fig. 15 is 4.13 on a five-point scale.

\section{Discussion and Future Work}

The beneficial effect of release adjustment was clearly demonstrated by the average number of completions, as well as in terms of the completion ratios. This was also confirmed by the subjective preferences.

In the preliminary experiment, the improvements enabled by the position and viewpoint adjustment methods were less clearly demonstrated, because only the completion ratios within a specified period were measured, because the adjustment model did not match the users' preference, and because the error tolerance, which is the difference between the target and control sphere sizes, was too large to clearly show the effects. The second experiment tried smaller error tolerance targets, used a stage model with hysteresis as an adjustment model, and measured the number of completions. The results of this experiment showed that the adjustments were effective for precise, efficient positioning and release.

In these experiments, we investigated the effect of automatic adjustments on only the translational positions of the virtual objects. We now plan to study the effect of automatic adjustments on the rotation of virtual objects.

Although experiments using direct hand manipulation without a handheld device were conducted, we believe that the methods tested in this research can also improve the efficiency of manipulation using a handheld device with a button. We will thus evaluate the adjustment methods for manipulation with a handheld device.

These experiments used fixed thresholds to simply demonstrate the effectiveness of the proposed adjustments. Some subjects commented, however, that they could not understand the effect of the position adjustment, and some did not like the viewpoint movement. These comments suggest that some parameters may have to be automatically customized for each subject. This requires further study.

\section{Conclusions}

The goal of this study was to develop an intuitive interface for use in an immersive virtual environment. This paper has described a system of enhanced hand manipulation to allow efficient, precise positioning and release of virtual objects through the use of automatic adjustment methods.

The proposed adjustment methods were evaluated experimentally. The results showed that, in terms of the number of completions, the completion ratio, and subjective evaluation, all of the methods were effective. Moreover, the results showed that release adjustment was the most important of the adjustment methods. The results also suggested that release adjustment was followed in importance by position adjustment, and then viewpoint adjustment with size adjustment.

These methods efficiently enable users to adjust the position of a virtual object both easily and precisely. This will give designers an additional way to increase the usability of hand manipulation in immersive virtual environments. Moreover, this would be useful to enable casual users to manipulate virtual objects effectively without extensive training.

\section{Acknowledgments}

We would like to thank the anonymous reviewers and the meta-reviewer for their valuable comments.

This research was partially supported by a Japanese Grant-in-Aid for Scientific Research (14380090, 19650251).

\section{References}

[1] K. Asai, N. Osawa, and Y. Sugimoto, "Virtual environment system on distance education," EUROMEDIA '99, pp.242-246, 1999.

[2] G. Barnes, "Visual programming agents for virtual environments," Proc. 2000 AAAI Spring Symp., pp.173-177, 2000.

[3] D. Bowman and L. Hodges, "An evaluation of techniques for grabbing and manipulating remote objects in immersive virtual environments," Proc. Symposium on Interactive 3D Graphics. ACM, pp.3538, 1997.

[4] B. Conner, S. Snibbe, P. Herndon, C. Robbins, C. Zeleznik, and A. van Dam, "Three-dimensional widgets," Proc. Interactive 3D Graphics Symposium, pp.183-188, 1992.

[5] S. Card, T. Moran, and A. Newell, The Psychology of HumanComputer Interaction, Lawrence Erlbaum, 1983.

[6] S. Frees and G.D. Kessler, "Precise and rapid interaction through scaled manipulation in immersive virtual environments," Proc. IEEE Virtual Reality 2005, pp.99-106, 2005.

[7] B. Fröhlich and J. Plate, "The cubic mouse: A new device for threedimensional input," Proc. CHI 2000 Conference on Human Factors in Computing Systems, pp.526-531, 2000.

[8] G. de Haan, M. Koutek, and F. Post, "IntenSelect: Using dy- 
namic object rating for assisting 3D object selection," Proc. 9th Int. Workshop on Immersive Projection Technology, 11th Eurographics Workshop on Virtual Environments (IPT/EGVE 2005), pp.201-209, 2005.

[9] R. Holm, E. Stauder, R. Wagner, M. Priglinger, and J. Volkert, "A combined immersive and desktop authoring tool for virtual environments," Proc. IEEE Virtual Reality Conference 2002, pp.93-100, 2002.

[10] T. Igarashi and K. Hinckley, "Speed-dependent automatic zooming for browsing large documents," Proc. 13th Annual ACM Symposium on User Interface Software and Technology (UIST2000), pp.139-148, 2000.

[11] H. Kim, G. Albuquerque, S. Havemann, and D.W. Fellner, "Tangible 3D: Hand gesture interaction for immersive 3D modeling," Proc. 9th Int. Workshop on Immersive Projection Technology, 11th Eurographics Workshop on Virtual Environments (IPT/EGVE 2005), Eurographics, pp.191-199, 2005.

[12] Y. Kitamura, S. Ogata, and F. Kishino, "A manipulation environment of virtual and real objects using a magnetic metaphor," Proc. ACM Symposium on Virtual Reality Software and Technology 2002, pp.201-207, 2002.

[13] G.A. Lee, G.J. Kim, and C.-M. Park, "Modeling virtual object behavior within virtual environment," Proc. ACM Symposium on Virtual Reality Software and Technology 2002, pp.41-48, ACM, 2002.

[14] M. Mine, F. Brooks, and C. Sequin, "Moving objects in space: Exploiting proprioception in virtual-environment interaction," Proc. ACM SIGGRAPH 97, pp.19-26, ACM, 1997.

[15] N. Osawa, K. Asai, and F. Saito, "An interactive toolkit library for 3D Applications: it3d," Proc. 8th Eurographics Workshop on Virtual Environments (EGVE2002), pp.149-157, 2002.

[16] N. Osawa and X. Ren, "Virtual 3D gearbox widget technique for precise adjustment by hand motion in immersive VR," IEICE Trans. Inf. \& Syst., vol.E87-D, no.10, pp.2408-2414, Oct. 2004.

[17] N. Osawa, "Enhanced hand manipulation for efficient and precise positioning and release," Proc. 9th Int. Workshop on Immersive Projection Technology, 11th Eurographics Workshop on Virtual Enviroments (IPT/EGVE 2005), Eurographics, pp.221-222, 2005.

[18] N. Osawa, "Automatic adjustments for efficient and precise positioning and release of virtual objects," Proc. ACM SIGGRAPH International Conference on Virtual Reality Continuum and Its Applications (VRCIA 2006), pp.121-128, 2006.

[19] J. Pierce, A. Forsberg, M. Conway, S. Hong, R.C. Zeleznik, and M. Mine, "Image plane interaction techniques in 3D immersive environments," Proc. Symposium on Interactive 3D Graphics, ACM, pp.39-43, 1997.

[20] I. Poupyrev, M. Billinghurst, S. Weghorst, and T. Ichikawa, "Gogo interaction technique: Non-linear mapping for direct manipulation in VR," Proc. ACM Symposium on User Interface Software and Technology (UIST'96), ACM, pp.79-80, 1996.

[21] M. Pinho, D. Bowman, and C. Freitas, "Cooperative object manipulation in immersive virtual environments: Framework and techniques," Proc. ACM Symposium on Virtual Reality Software and Technology 2002, pp.171-178, 2002.

[22] D. Thalmann, "Using virtual reality techniques in the animation process," in Virtual Reality Systems, ed. R. Earnshaw, M. Gigante, and H. Jones, pp. 143-159, Academic Press, 1993.

[23] N.M. Thalmann and D. Thalmann, "3-D devices and virtual reality in human animation," Proc. Computer Animation and Simulation '91, pp.169-181, 1991.

[24] S. Zhai, W. Buxton, and P. Milgram, "The partial occlusion effect: Utilizing semi-transparency in 3D human computer interaction," ACM Trans. Computer Human Interaction, vol.3, no.3, pp.254-284, 1996.

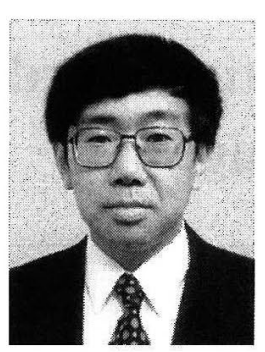

Noritaka Osawa received his B.S., M.S., and Ph.D. degrees in information science from the University of Tokyo in 1983, 1985, and 1988, respectively. After working for a software company and the University of Electrocommunications, he joined the National Institute of Multimedia Education in 1998. He was jointly appointed to the Graduate University for Advanced Studies in 2001 and is now a professor there. His current research interests include human-computer interfaces using virtual reality technologies, information visualization, and system software. 